\title{
COMPOSICIÓN FITOQUÍMICA DEL ACEITE DE LAS SEMILLAS DEL FRUTO DEL “AGUAYMANTO”, Physalis peruviana $L$.
}

\author{
Nancy Chasquibol Silva*1, Juan Carlos Yácono Llanos ${ }^{1}$
}

\begin{abstract}
RESUMEN
Se estudió el contenido de ácidos grasos y antioxidantes en el aceite de las semillas del fruto del "aguaymanto". La extracción del aceite crudo se realizó por el método de prensado por expeller con un rendimiento del 18,09\%. Se cuantificó ácidos grasos, tocoferoles y esteroles por métodos cromatográficos. El aceite presenta un alto porcentaje de $\omega-6(76,7 \%)$ con un grado de insaturación de 77,1 \%. Los tocoferoles $\alpha$ - (70 ppm), $\beta$ - (1452 ppm), $\gamma$ - (1469 $\mathrm{ppm})$ y $\delta$ - (1445 ppm) son las principales especies que han sido separadas y cuantificadas por HPLC. Los esteroles $\delta$-5-avenasterol $(17,1 \%), \beta$-sitosterol $(9,7 \%)$, estigmasterol $(4,9 \%)$ y campesterol $(4,8 \%)$, son las especies más importantes separadas y cuantificadas por CG. Los resultados obtenidos confirman que el aceite y la torta extraídos de las semillas del "aguaymanto" podrían ser considerados como una fuente dietética de antioxidantes naturales para promover una buena salud y cuidado de la piel.

Palabras clave: Ácidos grasos, semillas de aguaymanto, tocoferoles, esteroles

\section{PHYTOCHEMISTRY COMPOSITION OF OIL'S SEEDS FRUIT OF "AGUAYMANTO", Physalis peruviana $L$.}

\begin{abstract}
The content of fatty acids and antioxidants in the oil of the seeds of fruit of "aguaymanto" were studied. The extraction of crude oil was carried out by the expeller pressing method with a yield of $18.09 \%$. Fatty acids, tocopherols and sterols by chromatographic methods were quantified. The oil has a high percentage of $\omega-6(76.7 \%)$ with a degree of unsaturation of $77.1 \%$. The $\alpha$ - tocopherols (70 ppm), $\beta$ - (1452 ppm), $\gamma$ - (1469 ppm) and $\delta$ - (1445 ppm) are the main species have been separated and quantified by HPLC. Sterols $\delta$-5-avenasterol $(17.1 \%), \beta$-sitosterol $(9.7 \%)$, stigmasterol $(4.9 \%)$ and campesterol $(4.8 \%)$, are the most important species have been separated and quantified by GC. The results obtained confirm that the oil and cake extracted from the seeds of "aguaymanto" could be considerate as a source of natural antioxidants to promote good health and skin care.
\end{abstract}

Key words: Fatty acids, aguaymanto seeds, tocopherol, sterols

\section{INTRODUCCIÓN}

El aguaymanto es un fruto oriundo del Perú, descubierto en 1753 por el científico sueco Carlos Linneo, quien lo clasificaría como Physalis peruviana L. Existen diversas referencias históricas que indican fue domesticado, cultivado y muy apreciado por los antiguos peruanos.

${ }^{1}$ Facultad de Ingeniería Industrial, Instituto de Investigación Científica (IDIC) de la Universidad de Lima-Perú, Universidad de Lima, Av. Javier Prado Este, cuadra 46 s/n, Monterrico, Lima 33-Perú.- *nchsilva@ulima.edu.pe 
El "aguaymanto" posee diferentes nombres comunes: tomatito silvestre, capulí, uchuva, uvilla, cereza de los andes, awaymanto y bolsa de amor. Sus frutos son bayas carnosas, jugosas de color naranja-amarillo de forma globosa, con un diámetro entre 1,25 - 2,5 cm y contiene numerosas semillas. Los frutos presentan un sabor peculiar agridulce de buen gusto, protegidos por un cáliz no comestible de textura papirácea. El "aguaymanto" es una solanácea, pariente de la papa, tomate, ají y rocoto. Crece en la costa, sierra y selva del Perú, en los departamentos de Amazonas, Ancash, Ayacucho, Cajamarca y Cuzco; en suelos pobres, con bajos requerimientos de fertilización, pero bien drenados, y con gran luminosidad; debe protegerse del viento excesivo; además de contar con suficiente agua durante el desarrollo, más no en la maduración de sus frutos. Sus frutos son consumidos frescos o procesados en mermeladas, conservas, helado, yogures, pastelería, jugos y en la gastronomía peruana. Es un fruto con gran potencial económico y gran demanda en el extranjero, debido a sus propiedades nutricionales y medicinales ${ }^{1}$. La pulpa de la fruta contiene compuestos fenólicos, carotenoides, vitamina $\mathrm{E}$ y vitamina $\mathrm{C}^{2}$. El fruto contiene $15 \%$ de sólidos solubles, principalmente azúcares, con un alto contenido de fructuosa y que puede ser consumido por personas diabéticas ${ }^{3}$.

Los objetivos del presente trabajo fueron: seleccionar el método de extracción del aceite, estudiar la composición fitoquímica del aceite y la composición nutricional de la "torta" de las semillas del fruto del "aguaymanto".

\section{Obtención de las muestras}

\section{PARTE EXPERIMENTAL}

Los frutos del aguaymanto (Physalis peruviana L), fueron obtenidos en el "mercado mayorista de frutas" de la ciudad de Lima. La fruta lavada se despulpó para separar las semillas de la pulpa y piel mediante un procesador de jugos de frutas. Las semillas se lavaron con abundante agua y se secaron en la estufa a $40^{\circ} \mathrm{C}$ por un tiempo de 10 horas, para disminuir el contenido de humedad hasta un porcentaje inferior al $4 \%$ para evitar la degradación del color y el aumento de ácidos grasos libres.

\section{Extracción del aceite}

Con la finalidad de elegir el mejor proceso de extracción del aceite, se realizó pruebas experimentales con pequeñas cantidades de semillas seca, empleándose los siguientes métodos: extracción por Soxhlet, prensado tipo hidráulico y prensado tipo expeller. De los resultados obtenidos, se seleccionó el método de prensado por expeller (figura 1). Se empleó $710 \mathrm{~g}$ de semillas secas, separándose el aceite crudo y la "torta" para su estudio. El aceite se centrifugó a 4000 rpm, se almacenó en frasco oscuro y en refrigeración.

\section{Composición de ácidos grasos}

La identificación y determinación cuantitativa de los ácidos grasos, se realizó por CG después de la trans-esterificación del aceite con $\mathrm{KOH} 2 \mathrm{~N}$ en metanol de acuerdo al Método Estándar de la IUPAC ${ }^{4,5}$. El análisis cromatográfico se realizó con $2 \mu \mathrm{L}$ de muestra, empleando un CG Agilent 5890, equipado con un inyector Split, una columna capilar de sílice fundida 
SPB-2380 (60 m longitud x $0,25 \mathrm{~mm}$ de diámetro interno x $0,2 \mu \mathrm{m}$ de espesor de fase) y un detector de ionización de llama (FID). Se empleó gas portador de hidrógeno a una velocidad de flujo de $1,0 \mathrm{~mL} / \mathrm{min}$. Las temperaturas del inyector y del detector fueron de 275 y $300^{\circ} \mathrm{C}$, respectivamente. La temperatura inicial del horno fue de $165^{\circ} \mathrm{C}$ durante 15 minutos y con gradiente de temperatura de 165 a $200^{\circ} \mathrm{C}$ a $3^{\circ} \mathrm{C} / \mathrm{min}$.

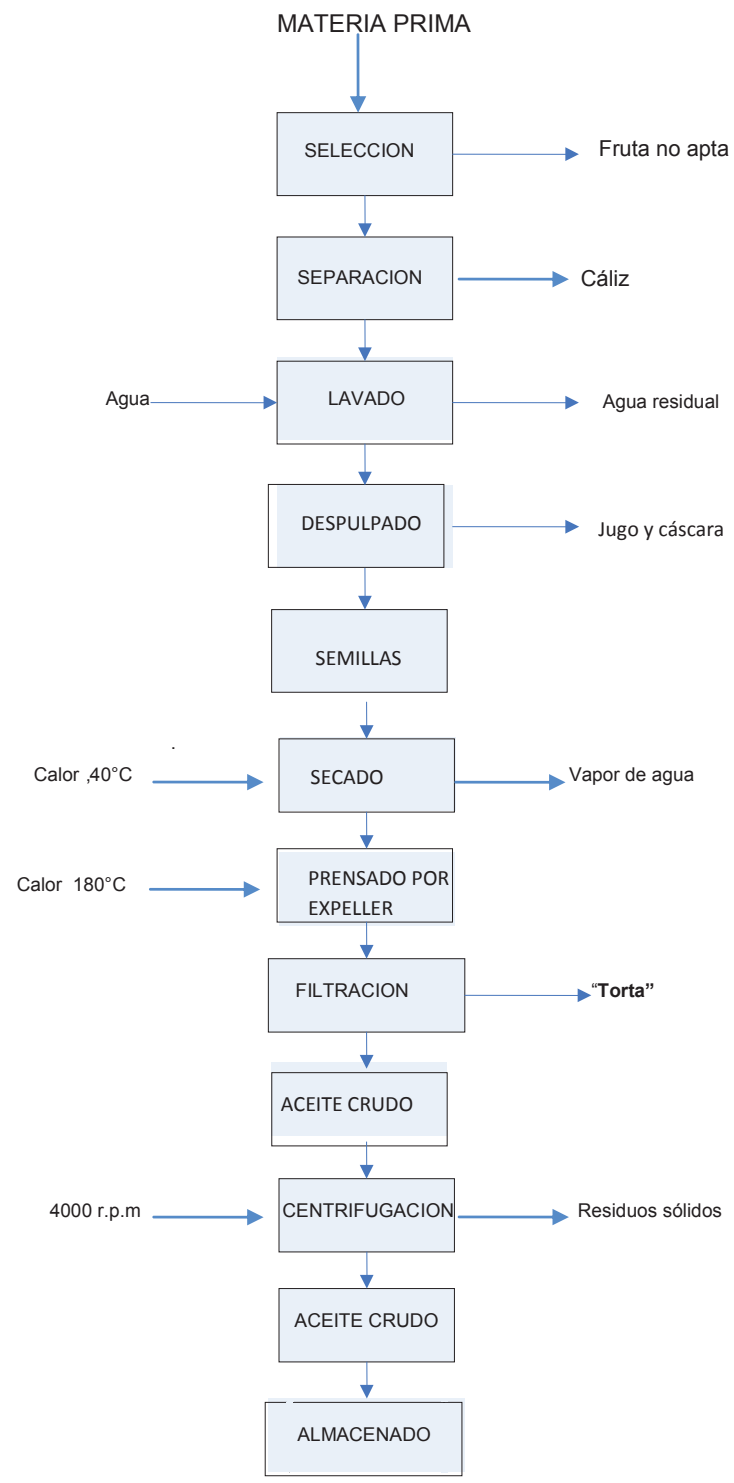

Figura 1. Diagrama de operación de procesos de extracción del aceito crudo de las semillas del fruto del "aguaymanto", Physalis peruviana L (Elaboración propia) 


\section{Tocoferoles}

Los tocoferoles fueron determinados de acuerdo al Método Estándar IUPAC $2432^{6}$. Se pesó $0,1000 \mathrm{~g}$ de aceite y se disolvió en $10 \mathrm{~mL}$ de n-hexano. Se empleó $20 \mu \mathrm{L}$ de la solución y se analizó por HPLC equipado con una columna lichrosorb SI 60 (250 mm longitud x $4 \mathrm{~mm}$ diámetro interno, $5 \mu \mathrm{m}$ de tamaño de partícula). La fase móvil fue n-hexano: 2-propanol ( $0,5 \%$, $\mathrm{v} / \mathrm{v}$ ) con una velocidad de flujo de $1 \mathrm{~mL} / \mathrm{min}$. La detección fue realizada por fluorescencia, con longitudes de onda de excitación y de emisión a $\lambda=290$ y $\lambda=330 \mathrm{~nm}$, respectivamente. La identificación y cuantificación de tocoferoles se llevó a cabo con patrones de $\alpha-, \beta-, \gamma-y$ $\delta$-tocoferoles.

\section{Composición de esteroles}

El análisis se realizó de acuerdo al Reglamento CEE/2568/91, del 5/9/1991, pág. $1^{7}$. El método consistió en obtener los compuestos saponificados con solución etanólica de $\mathrm{KOH} 2 \mathrm{~N}$. El compuesto insaponificado se fraccionó por cromatografía en capa fina de gel de sílice utilizando la mezcla de hexano/éter etílico $(65: 35, \mathrm{v} / \mathrm{v})$. La placa se reveló con 2,7-diclorofluoresceina y la banda de esteroles se separó con cloroformo y éter dietílico caliente. La solución se evaporó hasta sequedad, derivatizada con $500 \mu \mathrm{L}$ de la mezcla 1:3:9 (v/v/v) de trimetilcloroxilano:hexametildisilazano:piridina y analizada por CG. El cromatógrafo de gas se equipó con una columna capilar HP-5 de $25 \mathrm{~m}$ x 0,25 mm de diámetro interno x 0,2 $\mu \mathrm{m}$ de espesor de la fase. Se empleó como detector FID y el trabajo del horno se programó en régimen isotérmico a $260^{\circ}$, con gas portador de hidrógeno a una velocidad de flujo de $1 \mathrm{~mL} / \mathrm{min}$. La temperatura del inyector y detector fueron de $300^{\circ} \mathrm{C}$. La determinación cuantitativa fue realizada empleando colestanol como estándar interno.

\section{Análisis de la "torta"}

La composición nutricional de la "torta" de las semillas del fruto del "aguaymanto" en forma de hojuelas, se determinó en base a la humedad ${ }^{8}$, cenizas $^{9}$, proteínas $^{10}$, aceites y $\operatorname{grasas}^{11} \mathrm{y}$ fibra $^{12}$.

\section{RESULTADOS Y DISCUSIÓN}

El rendimiento del aceite por el método de prensado tipo expeller para un peso total de $710 \mathrm{~g}$ de semillas, fue de 18,09\% (tabla 1); se seleccionó este método porque no emplea solventes y porque desactiva las enzimas que favorecen la oxidación del aceite, la formación de olores y sabores desagradables; se obtuvo aceites y "tortas" de alta calidad, estabilidad y duración, con bajos niveles de fosfátidos y peróxidos. 
Tabla 1. Rendimiento del aceite crudo de las semillas del fruto del "aguaymanto"

\begin{tabular}{ccl}
\hline \multicolumn{1}{c}{ Método } & Peso de semillas (g) & \% Rendimiento \\
Prensado hidráulico & 713 & Gotas de aceite \\
Extracción por Soxhlet & 100 & 19,25 \\
Prensado por expeller & 710 & 18,09 \\
\hline
\end{tabular}

En el aceite crudo de las semillas del fruto del "aguaymanto", se identificó 14 ácidos grasos; el ácido graso predominante fue el ácido linoleico (76,7\%), seguido por el ácido oleico $(11,3 \%)$; ácidos grasos saturados (10,50\%); ácidos grasos monoinsaturados $(12,50 \%)$; dienos totales $(76,70 \%)$ y trienos totales $(0,30 \%)$ (tabla 2$)$. Estos resultados están de acuerdo a los datos reportados por Ramadan ${ }^{3}$. El ácido linoleico es considerado un ácido graso esencial muy importante para prevenir enfermedades cardiovasculares, arterioesclerosis e hipertensión. Los ácidos grasos poliinsaturados también son importantes en el mantenimiento de la membrana celular, en la producción de prostaglandinas, entre otros. Por lo que el consumo de estos aceites es importante porque también sirven como medio de transporte de las vitaminas liposolubles (A,D,E y K) y porque regulan el metabolismo del colesterol.

Tabla 2. Composición en ácidos grasos del aceite crudo de las semillas del fruto del "aguaymanto"

\begin{tabular}{|c|c|c|c|c|}
\hline & Compuesto & $\%$ & Compuesto & $\%$ \\
\hline C14:0 & (mirístico) & Trazas & Ácidos grasos saturados & 10,50 \\
\hline C16:0 & (palmítico) & 6,5 & Ácidos grasos monoinsaturados & 12,50 \\
\hline C16:1 & $\omega-7$ (palmitoleico) & 0,5 & Dienos totales & 76,70 \\
\hline $\mathrm{C} 17: 0$ & (margárico) & 0,1 & Trienos totales & 0,30 \\
\hline $\mathrm{C} 17: 1$ & (margaroleico) & Trazas & & \\
\hline C18:0 & (esteárico) & 3,1 & & \\
\hline C18:1 & $\omega-9 \quad$ (oleico) & 11,3 & & \\
\hline $\mathrm{C} 18: 1$ & $\omega-7$ (cis-vaccénico) & 0,6 & & \\
\hline $18: 2$ & $\omega-6 \quad$ (linoleico) & 76,7 & & \\
\hline $18: 3$ & $\omega$-3 (linolénico) & 0,3 & & \\
\hline C20:0 & (araquídico) & 0,4 & & \\
\hline $\mathrm{C} 20: 1$ & (eicosenoico) & 0,1 & & \\
\hline $\mathrm{C} 22: 0$ & (behénico) & 0,2 & & \\
\hline $\mathrm{C} 24: 0$ & (lignocérico) & 0,2 & & \\
\hline
\end{tabular}


Los tocoferoles $\alpha-, \beta-, \gamma-\mathrm{y} \delta$ - son las principales especies que han sido separadas y cuantificadas por HPLC (tabla 3 ). Los tocoferoles, $\gamma-, \beta$ - y $\delta$ - son las especies mayoritarias, mientras que para Ramadan $^{3}$, los tocoferoles $\beta$ - y $\gamma$ - son los componentes mayoritarios. Los tocoferoles son nutrientes esenciales; no son metabolizados por el cuerpo humano y por consiguiente deben ser incluidos en la dieta. Se han realizado muchos estudios para demostrar la importancia del $\alpha$-tocoferol en la protección de aceites de la peroxidación lipídica, en el daño del ADN, en la disfunción mitocondrial y en la disminución de la memoria y el aprendizaje. Existen también estudios que relacionan el lento deterioro cognitivo relacionado con la edad, por la ingesta diaria de vitamina E combinada con tocoferoles. Se ha estudiado también los efectos interactivos de los tocoferoles $\alpha$ - y $\gamma$-, demostrándose que el $\gamma$-tocoferol está relacionado con una menor neuropatología frente a la enfermedad de Alzheimer y que el consumo de dosis altas de $\alpha$-tocoferol no tiene un efecto neuroprotectivo ${ }^{13}$.

Tabla 3. composición de tocoferoles y esteroles del aceite crudo de las semillas del fruto del "aguaymanto"

\begin{tabular}{lclc}
\hline Tocoferoles & $\mathbf{m g} / \mathbf{K g})$ & Esteroles & $\%$ \\
$\alpha$-tocoferol & 70 & Campesterol & 4,8 \\
$\beta$-tocoferol & 1452 & Estigmasterol & 4,9 \\
$\gamma$-tocoferol & 1469 & $\beta$-sitosterol & 9,7 \\
$\delta$-tocoferol & 1445 & $\delta$-5-avenasterol & 17,1 \\
Tocoferoles totales & 4436 & $\delta$-5,24-estigmastadienol & 0,3 \\
& & $\delta$-7-avenasterol & 0,4 \\
& & Otros & 11,9 \\
& & Esteroles totales & $12696 \mathrm{mg} / \mathrm{Kg}$ \\
\hline
\end{tabular}

Los esteroles $\delta$-5-avenasterol $(17,1 \%), \beta$-sitosterol $(9,7 \%)$, estigmasterol $(4,9 \%)$ y campesterol $(4,8 \%$ ) (tabla 3$)$, son las especies más importantes cuantificadas por CG; mientras que para Ramadan $^{14}$, los esteroles campesterol y $\beta$-sitosterol son los componentes mayoritarios. La importancia del consumo de alimentos con alto contenido de esteroles está basado en evidencias científicas respecto al efecto hipocolesterolémico ${ }^{15}$, por lo que el consumo de esta fruta podría reducir los niveles de colesterol y prevenir enfermedades cardiovasculares, entre otros.

La composición química nutricional de la "torta" de las semillas del fruto del "aguaymanto" en forma de hojuelas indica buenas características nutricionales por el destacado aporte de carbohidratos, fibra, grasas, proteínas y cenizas (tabla 4). 
Tabla 4. Composición proximal de la "torta" de las semillas del fruto del "aguaymanto"

\begin{tabular}{lc}
\hline Composición proximal & $(\mathbf{\%})$ \\
Humedad & 3,92 \\
Cenizas & 2,84 \\
Proteínas & 14,58 \\
Carbohidratos & 32,51 \\
Grasas & 14,63 \\
Fibra & 31,52 \\
\hline
\end{tabular}

\section{CONCLUSIONES}

El método de prensado tipo expeller fue el método seleccionado para la extracción del aceite. El aceite extraído de las semillas del "aguaymanto" podría ser considerado una nueva fuente de aceite natural, por su alto grado de insaturación y contenido de tocoferoles y esteroles. Estos componentes bioactivos podrían servir como fuente dietética de antioxidantes naturales para prevenir enfermedades y promover una buena salud y cuidado de la piel. La "torta", debido a sus características nutricionales, podrían servir también para la elaboración de galletas, barras energéticas, entre otros.

\section{AGRADECIMIENTO}

Los autores expresamos nuestro agradecimiento a la Facultad de Ingeniería Industrial y al Instituto de Investigación Científica (IDIC) de la Universidad de Lima por el apoyo financiero y facilidades brindadas.

\section{BIBLIOGRAFÍA}

1. Hassanien, M. F. R. Physalis peruviana: a rich source of bioactive phytochemicals for functional foods and pharmaceutical. Food Reviews International. 2011; 27(3): 259273.

2. Ramadan, M. F., \& Moersel, J. T. Impact of enzymatic treatment on chemical composition, physicochemical properties and radical scavenging activity of goldenberry (Physalis peruviana L.) juice. Journal of the Science of Food and Agriculture. 2007; 87: 452-460.

3. Ramadan M. F. Bioactive phytochemicals, nutrition value and functional properties of cape gooseberry (Physalis peruviana L.): An overview. Food Research International . 2011; 44: 1830-1836. 
4. IUPAC Standard Method 2.301. Standard methods for the analysis of oils, fats and derivatives. Preparation of fatty acid methyl ester.Blackwell Scientific: Oxford, Great Britain, 1987

5. IUPAC Standard Method 2.302. Standard methods for the analysis of oils, fats and derivatives. Determination of FAMES by capillary GC. Blackwell Scientic: Oxford, Great Britain, 1987

6. IUPAC Standard Method 2.432.Standard methods for the analysis of oils, fats and derivatives . Determination of tocopherol and tocotrienols in vegetable oils and fats by HPLC. Blackwell Scientific:Oxford, Great Britain, 1987

7. Reglamento CEE/2568/91 (Diario Oficial de las Comunidades Europeas L248, del 5/9/1991, pág. 1 relativo a las características de los aceites de oliva y sobre sus métodos de análisis). Anexo 5: Determinación de la composición y del contenido en esteroles mediante $\mathrm{CG}$ con columna capilar.

8. AOAC. Official methods of analysis, Association of Official Analytical Chemists, $13^{\text {th }}$ ed., Washington DC, 1983

9. AOAC. Official methods of analysis, Association of Official Analytical Chemists, $15^{\text {th }}$ ed., Washington DC. 942.05, 1990.

10. AOAC. Official methods of analysis, Association of Official Analytical Chemists, $15^{\text {th }}$ ed., Washington DC, 920, 1990

11. AOAC. Official methods of analysis, Association of Official Analytical Chemists, $15^{\text {th }}$ ed., Washington DC. 981.11, 1990.

12. AOAC. Official methods of analysis, Association of Official Analytical Chemists, $15^{\text {th }}$ ed., Washington DC. 962.09, 1990.

13. Martha C. Morris, Julie A. Schneider, Hong Li, Christy C. Tangney, Sukriti Nag, David A. Bennett, William G. Honer, Lisa L. Barnes. Brain tocopherols related to Alzheimer's disease neuropathology in humans. Alzheimer's \& Dementia. 2015; 11: 32-39.

14. Ramadan and Morsel. Oil goldenberry (Physalis peruviana L.). Journal of Agricultural and Food Chemistry. 2003; 51(4): 969-974.

15. Helena Gylling, Jogchum Plat, Stephen Turley, and others, for the European Atherosclerosis Society Consensus Panel on Phytosterols. Plant sterols and plant stanols in the management of dyslipidaemia and prevention of cardiovascular disease. Atherosclerosis. 2014; 232: 346-360. 